\title{
Dissimilar metal joint quality measurement using infrared thermography: Experimental and Numerical approach for the application to CMT welding
}

\author{
by Renil Thomas K*, Sreedhar Unnikrishnakurup**, Krishnan Balasubramaniam, Lakshmi Narayanan**, \\ Phanikumar $\mathrm{G}^{\star \star * *}$. \\ * Centre for Non-destructive Evaluation, Mechanical Engineering Department, \\ Indian Institute of Technology Madras, Chennai, ktrenil@gmail.com. \\ ${ }^{*}$ Federal Institute for Material Research and Testing (BAM), Berlin. \\ *** Mechanical Engineering Department, Indian Institute of Technology Madras, Chennai. \\ ****Metallurgical and Materials Engineering Department, Indian Institute of Technology Madras, Chennai.
}

\begin{abstract}
Joining of dissimilar material has become highly popular research subject in the automobile industry due to the reduced weight and thereby increasing the fuel efficiency. Infrared thermography can be used as a natural tool to measure the temperature near the welding region and correlate the distribution of temperature to the weld quality. In the present work the quality of the dissimilar welded sample is identified using the temperature distribution in the vicinity of the weld pool region. A numerical model for CMT continues welding process has been modeled and simulated for the first time and compared with the experimental measurement.
\end{abstract}

\section{Introduction}

Mass reduction is the primary objective to achieve for the automotive industry for more fuel efficient vehicles in order to reduce the $\mathrm{CO}_{2}$ emissions. The replacement of the high density steel by the high strength and low mass density aluminum alloys is an emerging approach to achieve this goal. This approach encounter issues of joining aluminum alloys with the galvanized steel components in the automotive industry. Cold Metal Transfer (CMT) process is a recent development in welding technology which is suitable for the joining of aluminium and steel thin sheets.

CMT is a modified Metal Inert Gas (MIG) welding process which was invented by Fronius company. It combines both electrical and mechanical processes to achieve low heat input joining process which suits our application perfectly. There is a great interest in quantitatively defining the weld joint quality and thus optimizing the weld characteristics. Arc misalignment, plate mismatch, identification of weld pool impurities, effect of current on temperature for stationary arc and moving arc cases have been reported earlier [1]. Relationships have been established between peak surface temperature and depth of penetration and also between infrared line profile width and weld bead width during Gas Metal Arc and Gas Tungsten Arc welding processes [2]. The complete understanding of different physical phenomena occurring during the CMT welding process is not possible only with the experimental methods. With the advances in the computational resources, the numerical modeling can help in better understanding of the basic mechanisms. Over past few decades a lot of researchers developed models to study the welding process [3]

In the present work, a 3D heat diffusion phenomenon is modeled during the CMT welding of aluminum and galvanized steel. Experimental investigations were carried out using infrared thermographic measurement of the surface temperature during the welding cycle. Different cases of lack of joint formation were identified. The effect of this defect is incorporated in the simulation by adding a special boundary condition between the material contact sections. The developed model then validated with the different defective cases. This study provides an insight into the ways to incorporate defects in a numerical model during welding process modeling.

\section{Experimental procedure}

The lap joint configuration was fabricated from $100 \mathrm{mmX100mm}$ sheets. The Al sheet was placed on top of the $\mathrm{Zn}$ coated steel in a lap configuration with an overlap distance of $10 \mathrm{~mm}$. The welding direction is parallel to the lap seam. The CMT joining process is realized by use of a Fronius arc welding system. Al wire with a diameter of $1.2 \mathrm{~mm}$ was used in this study. Argon shielding gas with a flow rate of $16.5 \mathrm{l} / \mathrm{min}$ was used throughout the experiments. Infrared camera of medium wavelength range was used for the data acquisition with a frame rate of $150 \mathrm{~Hz}$. The camera was mounted on a tripod focusing on the entire lap configuration from top side of the weld. The temperature during the joining process is very high $\left(>1000^{\circ} \mathrm{C}\right)$ which is beyond the calibrated range of the present camera. But the objective of the present inspection is to look at the region away from the weld bead where the surface temperature of the plates fall in the camera's calibrated temperature range. Two columns of pixels were considered parallel to the weld bead with a distance of $10 \mathrm{~mm}$ away on the either sides of the joint. These columns contain the line surface temperature information on aluminium and steel. Temperature history of each pixel in the line is plotted for two columns. This data is compared for all produced welds 


\subsection{1/qirt.2016.056}

\section{Mathematical model}

The energy balancing equation that governs the distribution of temperature field can be expressed as follows.

$$
\rho C \frac{\partial T}{\partial t}=\nabla \cdot(k \nabla T)-\rho C u \cdot \nabla T+Q_{G}
$$

Where $\rho$ is the density, $C$ is the specific heat, $T$ is the temperature, $t$ is time, $k$ is the thermal conductivity, $u$ is the welding velocity, and $Q_{G}$ is the rate of heat generation or consumption per unit volume. The initial and boundary conditions imposed for the welding problem are:

$$
\begin{gathered}
T(x, y, z, 0)=T_{0}(x, y, z) \\
k_{n} \frac{\partial T}{\partial n}+q+h_{c}\left(T-T_{0}\right)+\sigma \varepsilon\left(T^{4}-T_{0}^{4}\right)=0
\end{gathered}
$$

Where $k_{n}$ is thermal conductivity normal to the surface, $h_{c}$ is the convective heat transfer coefficient, $T_{0}$ is the atmospheric temperature, $\sigma$ is the Stefan-Boltzmann constant, and $\varepsilon$ is the radiation emissivity.

The moving heat source in the $x$ direction is modeled with a constant velocity $u$. The heat source is modeled as a boundary condition on the welding surface. Different regions in the welded samples were identified as completely welded region and no-weld region. A continuous heat flux boundary condition is used for the completely weld region and thin thermally resistive layer (TTRL) boundary condition is used for the no-weld region. The finite element software package COMSOL Multiphysics is used to numerically solve the problem.

\section{Results and conclusion}

In this study, we tried to correlate the thermal cycles measured in the base metal which are away from the weld bead with the weld joint quality. Thermography provides an accurate estimation temperature over the entire surface. In addition to that a 3D transient heat conduction model has been developed to simulate the thermal cycles during CMT welding of aluminum and galvanized stainless steel. The thermal cycles in the base material which are measured away from the weld bead can provide qualitative information about the welding process. The temperature distribution on the aluminum base metal (figure 1) shows variation in welded region and non-welded region.

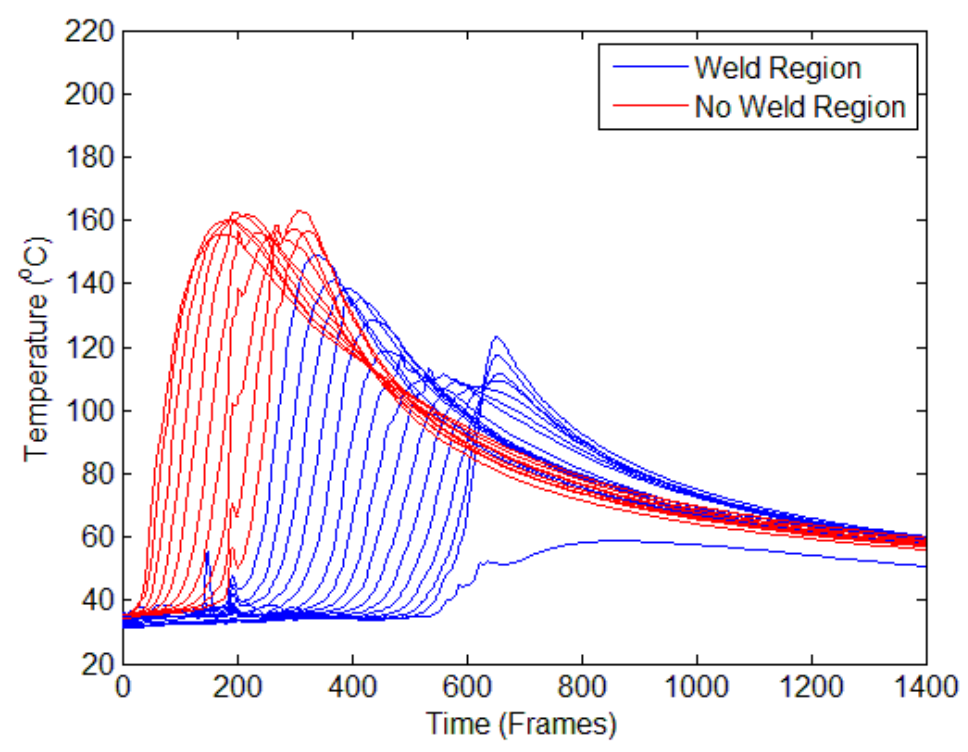

Fig. 1. Temperature distribution on the Al plate

\section{REFERENCES}

[1] N. H. Chin, B A Madsen and J. S. Goodlinc, "Infrared thermography for sensing the arc welding process," 1983.

[2] Y. Wang and B. A. Chin, "On line sensing of weld penetration using infrared themography," in NDT \& E International, 1986, vol. 29, no. 6, pp. 314-320.

[3] Z. Rao, J. Liu, P.-C. Wang, Y. Li, and S. Liao, "Modeling of Cold Metal Transfer Spot Welding of AA6061-T6 Aluminum Alloy and Galvanized Mild Steel," J. Manuf. Sci. Eng., vol. 136, no. 5, p. 051001, Aug. 2014. 